\title{
A critical review of development and demulsification mechanisms of crude oil emulsion in the petroleum industry
}

\author{
Sofiah Atirah Raya ${ }^{1}$ - Ismail Mohd Saaid ${ }^{1}$. Abdelazim Abbas Ahmed ${ }^{1} \cdot$ Abubakar Abubakar Umar $^{1}$
}

Received: 9 October 2019 / Accepted: 3 January 2020 / Published online: 14 January 2020

(c) The Author(s) 2020

\begin{abstract}
The need for efficient demulsification process to treat emulsions in the petroleum industry is well acknowledged. For decades, numerous researches have been conducted to examine mechanisms of emulsification and demulsification. Untreated emulsion has both technical and commercial implications in the industry, especially in terms of treatment facilities, refining and transportation. Effective treatment is needed to ensure optimum production of hydrocarbons. The present paper is to review reported works on the formation of petroleum emulsions, demulsification treatments, characteristics of fit-for-purpose demulsifiers as well as research trends in emulsion treatment. Crude oils are naturally combined with natural surfactants having high tendency to form stable emulsion. The stable emulsion must be treated well to meet industrial requirements since crudes with a high volume of stable emulsion have a less value. Therefore, fundamental studies on natural surfactants, which contribute to the emulsion stability, are analyzed for the effective separation of emulsions into oil and water. This would involve the assessment of various reported mechanisms for the emulsification and right formulation for effective demulsification.
\end{abstract}

Keywords Demulsification $\cdot$ Crude oil emulsion $\cdot$ Water-in-oil emulsion $\cdot$ Oil-in-water emulsion $\cdot$ Demulsifier

\section{Introduction}

Crude oil is found in association with gas and saline formation water. As the reservoir becomes depleted, water is coproduced with oil and the number of wells producing water with crude oil is steadily increasing. These immiscible fluids are readily emulsified by the simultaneous action of shear and pressure drop at the wellhead, chokes and valves (Matijasevic and Banhart 2006). When at least two immiscible phases are present in a system, they are called dispersion. The formation of a dispersed system involves a dispersed phase and a continuous flow (Goodarzi and Zendehboudi 2019).

Sofiah Atirah Raya

sofiahatirah@gmail.com

Ismail Mohd Saaid

ismailsaaid@utp.edu.my

Abdelazim Abbas Ahmed

abdelazim_g02667@utp.edu.my

Abubakar Abubakar Umar

hidima5050@gmail.com

1 Department of Petroleum Engineering, Universiti Teknologi PETRONAS, 32610 Bandar Seri Iskandar, Perak, Malaysia
Emulsion is a two-phase system comprising of two liquids not forming homogenous when mixed, one is (dispersed phase) constantly dispersed as globules in the second phase (continuous phase) (Mohyaldinn et al. 2018). During the extraction and transportation of crude oil, the formation of an emulsion is undeniable. The formation happens once the heterogonous mixture flows in the piping valves and porous rocks and endures turbulence at high temperature or high pressure. The primary reasons that enhanced the emulsion formation include the existence of surface-active agents, ionic compositions and $\mathrm{pH}$ of the water (Sjöblom 2001).

The stability of the crude oil emulsions has gained concerns among many oilfield researchers to invent different effective and relevant techniques to break it. Demulsification has expanded in significance because the usage of steam and caustic injection or combustion processes, for in situ recovery of heavy oils, is intricate by the development of viscous emulsions of oil, water and clay (Matijasevic and Banhart 2006).

The origin of emulsion which occurs from the oilfield reservoir is a complex problem in the petroleum industry. The tightening of future needs for the best quality of petroleum requires impressive and intense development efforts concerning to improve the demulsification mechanisms 
of the crude oil emulsions. According to Kokal and Aramco (2005), crude oil is rarely produced alone. It usually produced with water, which creates several complications during its production. There are two ways the water may produce. The water may be produced as free water, which will settle out immediately or the presence of water may lead to the formation of emulsion. The production of the emulsions is influenced by several factors (Zolfaghari et al. 2016; Sheng 2010):

- The addition of emulsifying agents at the oil-water interfaces such as surfactants, fine solids, organic or inorganic materials.

- The coproduct of water phase along with the production of crude oil.

- The pressure drops issuing during the pumping process.

- The flow of crude oil through permeable media.

The emulsions are reviewed as special liquid-in-liquid colloidal dispersions. The kinetic stability of the emulsions depends on their droplet size and the existence of interfacial films that occupied the water droplets. The presence of the stabilizing agents is also recognized as emulsifiers that are added or that occurred naturally during the crude oil production enabling to promote the emulsion kinetic stability. Consequently, emulsifiers such as asphaltenes and resins lead to flocculation and coalescence during emulsion breakdown. This formation of the emulsion and the mechanisms to break the emulsions is the major concern in this review.

\section{The emulsion issues}

Emulsions, which resulted naturally during the extraction of crude oil from the wellbore, are defined as a thermodynamically unstable system, coproduct with contamination of water, sediments and/or brine of different salts, and consist of two liquid phases (Biniaz et al. 2016; Umar et al. 2018) Kokal and Aramco (2005) stated that crude oil emulsions are recognized as a dispersion of water droplets in oil. The emulsions are unwelcomed to the transporter and producer because it has high possibility to produce a stable composition if not treated well, which leads to many difficulties, especially in the refining process operation. Many struggles may assemble during the extraction of crude oil from the petroleum well to be listed (Zolfaghari et al. 2016; Kokal and Aramco 2005):

- The formation of high pressure drops in flowlines.

- The increase in pumping and transporting cost of the water-oil mixture through the pumps and pipes.

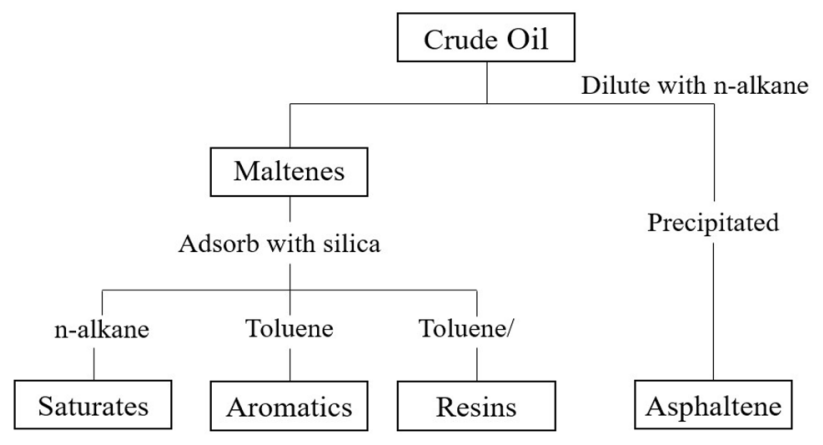

Fig. 1 The schematic of separating crude oils into SARA components (Auflem 2002)

- The destruction of pipeline and production amenities (due to two-phase flow and presence of chloride ions in the aqueous phase).

- The escalation in corrosion rate and scaling (due to the salt content in the formation of water).

- The tripping of separation equipment in gas/oil separating plants. (GOSPs) which limit the export quality of crude oil.

- The lowering of the oil API gravity.

- The destroying of downstream processing plant catalysts.

- The adversity involved to improve the viscosity of the crude oil (due to small dispersed water drops).

Consequently, the variety of problems caused by stable emulsion and the factors either enhance or reduce emulsion stability became the major attention in the petroleum industry.

When water-in-oil emulsions form, the physical properties of oil change comprehensively. For instance, from 60 to $80 \%$ water is involved in the stable emulsions, and thus, the spilled material grows from 2 to 5 times the original volume. Most prominently, the viscosity of the oil typically changes from a few hundred to about 100,000 mPas, an increment by a factor of 500-1000 (Fingas and Fieldhouse 2009). A liquid product is altered into a heavy, semisolid material. It was inferred that the thick emulsions are demanding supplementary tackle to be treated with ordinary spill recovery equipment (Goni et al. 2015).

\section{Classification of crude oil emulsions}

Crude oils can be categorized into four primary components: saturates (including waxes), aromatics, resins and asphaltenes which are called SARA fractionation. This classification of the crude oils is depending on its polarity and solubility in a solvent. Asphaltenes, which are separated as one of the groups, are a significant aspect of fluid 
Table 1 The properties of crude oil and their general relationship to sediment formation (Speight 1982)

\begin{tabular}{ll}
\hline Properties & Remarks \\
\hline Asphaltene content & Affects light oil recovery and heavy oil recovery \\
& Influences oil-rock interactions \\
& Forms sediment when gases are dissolved in the oil \\
& Thermal methods can change the relationship to oil \\
& Greater than 1.0: oil is stable \\
Asphaltene/resin ratio & Less than 1.0: oil is unstable \\
Heteroatom content & Offers polarity, causing incompatibility \\
& Preferential reaction with oxygen \\
Aromaticity & Responsible for incompatibility in paraffinic environments \\
API gravity & Small gravity, large asphaltene content \\
Viscosity & Large viscosity, large asphaltene content \\
Oil medium & Can be altered by thermal recovery processes \\
Oxidation & Changes in functional group composition \\
\hline
\end{tabular}

processability. The structure of the SARA fractionation is illustrated in Fig. 1.

The basic way to eliminate the asphaltenes is by precipitation in a paraffinic solvent. Meanwhile, the deasphalted oil (saturates, aromatics and resins) is separated by chromatographic fractionation. Out of the four classes, only saturates can easily be differentiable from the rest of hydrocarbons in the mixture. The absence of $\pi$-bonds affects them to readily discern the aromatic fractions by advantage of the difference in their polarity. The remaining oil is comprised of aromatics and heteroatomic compounds with different degrees of condensation, alkyl substitution and functionalism (Auflem 2002).

Also, the properties of the crude oil and their general relationship to sediment formation are tabulated in Table 1.

\section{Types of emulsion in the industry}

Two main types of emulsion, which regularly occur in the oil industry, are water-in-oil emulsions (where water phase dispersed in the continuous oil phase) and oil-in-water emulsions (where oil phase dispersed in the continuous water phase). These types of emulsion are thermodynamically unstable but at the same time kinetically stable because they may be lasting forever or even for a long time (Zolfaghari et al. 2016). Based on their kinetic stability, emulsions are categorized into three classes: loose, medium and tight emulsions. They differ from each other by their separation rate where loose emulsions will separate within a few minutes and the removed water at times discussed as free water. Medium emulsions will separate around in $10 \mathrm{~min}$. However, tight emulsions take longer time to separate as such for days, weeks or even not entirely separate.

The characterization of crude oil emulsions is shown in Figs. 2 and 3. Figure 2 shows the droplet size distribution

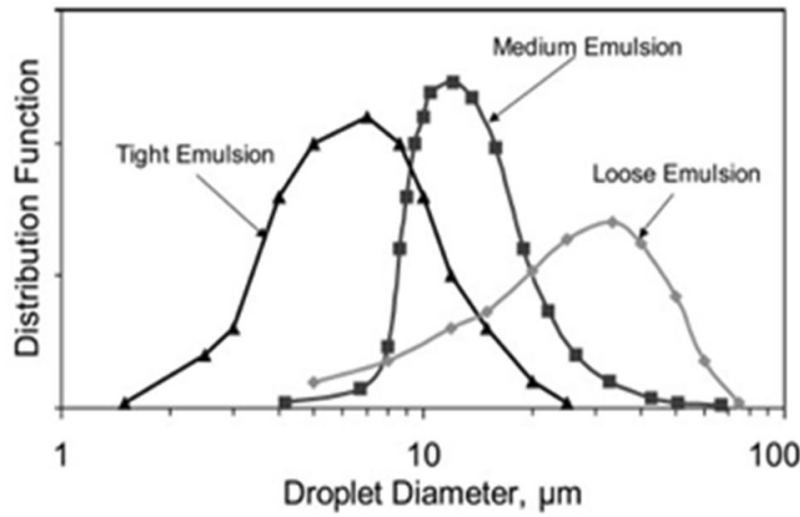

Fig. 2 Distribution function against droplet diameter (Kokal and Aramco 2005)

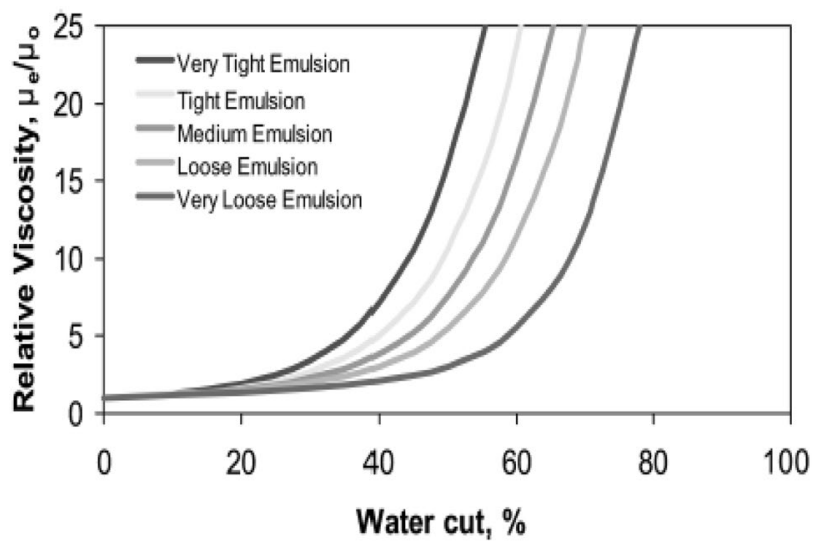

Fig. 3 Relative viscosity against water cut, \% (Kokal and Aramco 2005) 
of petroleum emulsions. Several factors such as IFT, shears, nature of emulsifying agents, existence of solids and bulk properties of oil and water influence the droplet size distribution. The stability of the emulsion is attained by the droplet size distribution. As a rule of thumb, small average size of dispersed water droplets increases the time of residence, and thus, a bigger size of separating plant equipment is needed. Figure 3 shows the relative viscosity that depends on the percentage of the water cut. The relative viscosity starts to increase dramatically at $40 \%$ and $60 \%$ for very tight emulsion and very loose emulsion, respectively. However, waterin-oil-in-water emulsions that are also known as multiple emulsions are applicable in foods, cosmetics, pharmaceutics and wastewater treatment where the inner aqueous phase is the dispersed phase of oil droplets (Zolfaghari et al. 2016).

\section{Water-in-oil emulsions}

The study of water-in-oil (W/O) emulsions behavior is the main attention because it usually exists during the exploration and extraction of crude oil (Wong et al. 2015). According to Zolfaghari et al. (2016), water-in-oil emulsions allocated the largest interest during the production of crude oil. These emulsions need to be separated into two phases to fulfill the needs of crude oil for transportation or need to be sent to a refinery. Water-in-oil emulsion (often named as "chocolate mousse" or "mouse") is commonly known as regular, while the oil-in-water emulsion is known as inverse or reverse. About $95 \%$ of the crude oil in the world produced the water-in-oil emulsion type (Sjöblom 2001). Zolfaghari et al. (2016) reviewed the process of water-in-oil emulsion formed. As discussed earlier, crude oil consists of two immiscible liquid phases: oil and water. It tends to separate into water droplet formation which dispersed in all through the continuous oil phase. The evolution of water-in-crudeoil emulsions is illustrated in Fig. 4. During production, an adequate mixing or presence of emulsifiers/surfactants added to the volume of the crude oil leads to corrosion in

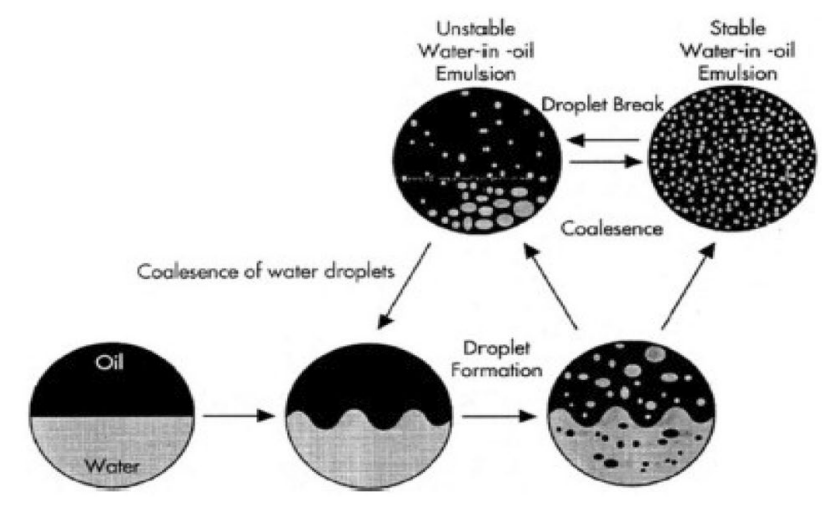

Fig. 4 The evolution of water-in-oil emulsion (Wong et al. 2015) the pipelines and growing transportation and refining cost and promotes the formation of stable emulsions. One of the important parameters during the transportation is the crude oil viscosity. The emulsion viscosity is affected by the droplet size distribution because the smaller the droplet size of the droplet, the higher the viscosity and stability of the water-in-oil emulsions. Thus, these emulsions need to be treated to reduce the problems associated during transportation in the production facilities (Goodarzi and Zendehboudi 2019; Lee 1999).

The water-in-oil emulsion will be formed when certain crude oils mixed with seawater (which have their natural brine, $\mathrm{NaCl}$ ) and produced droplets of water dispersed in the oil. Mixing energy that required to form emulsions is provided in the ocean by wind or wave turbulence (Lee 1999). Further discussion on the water-in-oil emulsion was proposed by Davies et al. (1996), Sjöblom (2001). They found several factors that discharge the limitation in the characterization of the emulsion. As such, water-in-oil emulsions contain a high concentration of water, numerous solids at the interphase and organic nature of the continuous phase. The size of the droplets and the rate of separation variables also play an important role to break the emulsion because the performance strongly relies upon them.

\section{Oil-in-water emulsions}

Oil-in-water $(\mathrm{O} / \mathrm{W})$ emulsions are said as "reverse" emulsions. $\mathrm{O} / \mathrm{W}$ emulsions are usually identified when oil droplets dispersed in the water phase. In 1994 (Porter 1991), Porter disclosed that the stabilization and adsorption of a surfactant in an emulsion are more effective when the surfactant is more soluble in the continuous phase. In the case of $\mathrm{O} / \mathrm{W}$ emulsion formation, it consists of two phases: water and oil. The oil phase appeared as globules in a continuous water phase, and by considering the structure of the surfactant (hydrophilic head and hydrophobic tail), the suitable surfactants are oil surfactant soluble type. However, for W/O emulsions, water surfactant type is more effective. Figure 5 shows the schematic illustration of $(\mathrm{W} / \mathrm{O})$ and $(\mathrm{O} / \mathrm{W})$ emulsions.

\section{Multiple emulsions}

The structure of multiple emulsions is more complicated and contains small droplets suspended in bigger droplets that are suspended in a continuous phase. For instance, water-in-oil-in-water emulsions comprise tiny water droplets suspended in larger oil droplets that are suspended in a continuous water phase (Kokal and Aramco 2005). This finding is supported by Israelachvili (1994), Sjöblom (2001) who claimed that multiple emulsions can be divided into two classes: water-in-oil-in-water $(\mathrm{W} / \mathrm{O} / \mathrm{W})$ emulsions and 


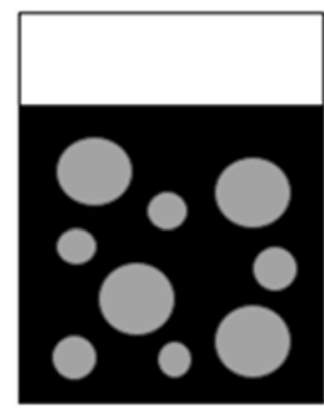

(a)

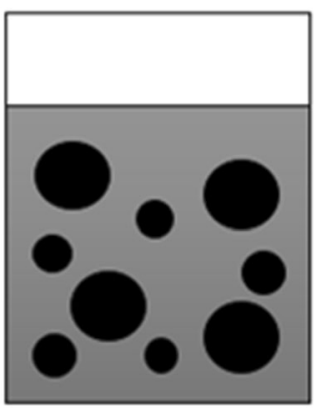

(b)
Fig. 5 A schematic illustration of water-in-oil (a) and oil-in-water emulsions (b)

oil-in-water-in-oil (O/W/O) emulsions. (W/O/W) emulsions are comprised of oil globules in water droplets that dispersed in water. Meanwhile, $(\mathrm{O} / \mathrm{W} / \mathrm{O})$ emulsions are comprised of water globules in oil droplets that dispersed in oil. These multiple emulsions may be the intermediate state when simple emulsions experience the changes from $\mathrm{W} / \mathrm{O}$ emulsions to $\mathrm{O} / \mathrm{W}$ emulsions.

The type of emulsion either water-in-oil or oil-in-water also can be identified by the type of emulsifying agents involved. Asphaltenes, resins, waxes, inorganic solids and oil-soluble organic acids are the surface-active agents that may produce along with the interfacial films that inhibit the coalescence process of the water droplets. The emulsifying agents are fundamentally established by hydrogen bonding of the $\mathrm{N}-, \mathrm{O}$, and $\mathrm{S}$ - comprising the groups existing in the crude oil besides $\mathrm{Si}-\mathrm{OH}$ and $\mathrm{Si}-\mathrm{O}-$ in dispersed materials at the oil and water combination (Zolfaghari et al. 2016; McLean and Kilpatrick 1997) To identify the formation of water-in-oil emulsion, the emulsifying agent is in the preference wetted by the oil phase, and the contact angle, between the oil-water-solid boundary, $\theta$ is more than $90^{\circ}$. Despite recognizing the formation of an oil-in-water emulsion, the emulsifying agent is in the preference wetted by the water phase and $\theta$ is less than $90^{\circ}$. When the contact angle $\theta$ is close to $90^{\circ}$, the formation of stable emulsions can be determined (Umar et al. 2018; Bobra 1991).

The stable emulsions sometimes fall between macroemulsions and microemulsions. By considering the droplet size, additional type of emulsion named macroemulsions (having dispersed phase diameters of greater than $0.1 \mu \mathrm{m}$ ) is said to be thermodynamically unstable structures because the contact between the oil and water molecules is undesirable so they will continuously break as a function of time (Umar et al. 2018). Moreover, different from its formation and stability, microemulsions as the second class of emulsion are introduced. They formed naturally when two immiscible water and oil phases with dramatically ultra-low interfacial energy are composed as one. Microemulsions are thermodynamically stable (unlike stable emulsions) and have dispersed phase diameters of less than $10 \mathrm{~nm}$. The small size of the droplets indicated microemulsions as a clear and translucent solution; also, from time to time the emulsions will break into separated water and oil phases (Fink 2012).

\section{Types of water-in-oil emulsions}

When water is mixed with the crude oil, they will form a water-in-oil emulsion that can be further categorized into four classes. They are stable water-in-oil emulsions, mesostable water-in-oil emulsions, entrained water and unstable water-in-oil emulsions (Fingas and Fieldhouse 2004). The studies of these types of emulsion are based on their stability caused by asphaltenes, resin, density, viscosity of the initial oil, elasticity, rheological properties, and as well as their visual appearance from greater than 400 oil samples and petroleum products. Among the four states, only stable and mesostable water-in-oil emulsions are treated as emulsions. Water content in the water-in-oil mixture is not primarily significant in this classification of emulsions (Wong et al. 2015; Fingas and Fieldhouse 2004). The same researcher also proposed numerical oil-spill modeling based on new empirical data and the corresponding physical knowledge of the emulsion formation.

Water-in-oil mixtures can be classified either stable, mesostable, entrained water or unstable water-in-oil emulsions (Wong et al. 2015). They are different in terms of spontaneous demulsification time under the laboratory condition, stability and appearance. They found that stable emulsions are always found in the oil and gas industry. Stable waterin-oil emulsions have the appearance of brown or reddish semisolid materials. They remain not to be separated into water and free oil for more than a week and remain whole within 4 weeks at least. Mesostable water-in-oil emulsions are broken into the water and free oil around one to 3 days. They seem to appear as black or brown liquid and assumed to be between stable and unstable emulsions. Next, as stated earlier entrained water and unstable water-in-oil emulsions are not considered as emulsions. The initial average water content of entrained water is around $40 \%$, which may be decreased to $15 \%$ in a week. They also disclosed that entrained water remains whole for less than a day. It appeared as a black liquid. Unstable water-in-oil emulsions are categorized as the oil that does not welcome a significant amount of water, and if it does, the water will remain in a very limited time.

According to Lee (1999), a stable emulsion consists of an aqueous phase, an oil phase and an emulsifying agent. The emulsifying agent that exists at the oil interphase is commonly known as particles or surfactants and facilitates the formation of a stabilized water-in-oil emulsion.

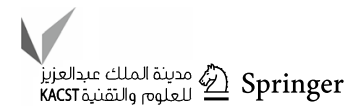




\section{Stabilization mechanism of w/o emulsions}

About 40 years ago, the formation of water-in-oil emulsions was renowned in the oil industry (Fingas 1995). Many oilfield researchers investigated that the emulsions are accompanied by some native solids, surfactants or functional molecules such as asphaltenes and resins which are contributed to the stabilized emulsion. Among the leading studies about the water-in-oil emulsions, according to Fingas and Fieldhouse (2004), Fingas (1995) and Goni et al. (2015), stability is the most significant behavior of its formation. Their investigation also showed that the content of asphaltene and resins is the major fraction that leads to emulsion stability.

According to Czarnecki and Moran (2005) and Czarnecki (2009), emulsion stabilization must contain a small fraction of asphaltenes and another surfactant material and further studies on the surface interaction were performed to determine the molecules that are responsible for the high stability of the water-in-oil emulsion. Figure 6 shows the formation of emulsions that are stabilized by asphaltenes. The presence of surfactants in the oil-water interface facilitates the development of small droplets which are significant in the preparation of emulsion. These surfactants enable to reduce the interfacial tension by assuming the surface free energy changes during emulsification. The main function of surfactants is to promote the formation of emulsion, generate a smaller droplet and aid the emulsion stability. A piece of fundamental knowledge about the emulsifiers can present an innovative solution to distinguish the formation of a stable emulsion.

It is generally known that four major elements which are saturates, aromatics, resins and asphaltenes can be found in the crude oils. They are varied from one another sufficiently in solubility and adsorptive appeal, and the relevant method can be applied to separate them from the crude oils. McLean and Kilpatrick (1997) had carried out a study on the effects of asphaltenes solvency on the stability of water-in-crude-oil emulsions. They found that the emulsion stability is mainly
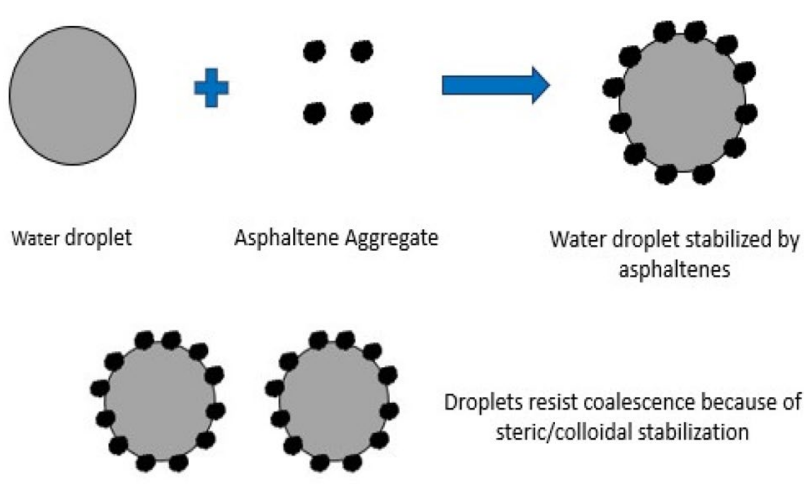

Fig. 6 Emulsions stabilized by asphaltenes (Kokal and Aramco 2005) supported by the state of the solubility of the asphaltenes in the crudes. Several factors affect the state of solubility of the asphaltenes, which include the characteristic of the crude oils, resin-to-asphaltene ratio, the aromaticity ratios of the crude medium and resins concerning the asphaltenes, and the concentration of polar functional groups in the resins and asphaltenes. Zaki (2000) claimed that the higher the concentration of the asphaltenes, the greater the emulsion stability. However, resins are not able to stabilize the emulsions without the existence of asphaltenes. This means that both asphaltenes and resins governed primarily the production of stable emulsions.

Asphaltenes are operationally defined as the nonvolatile and polar fraction of petroleum that is insoluble in n-alkanes (i.e., pentane or heptane). As a result, asphaltenes constitute a solubility class of crude oil components, rather than a chemical class. The molecular weight, polarity and aromaticity of precipitated asphaltenes generally increase with an increasing carbon number of n-alkane precipitant (Auflem 2002). Figure 7 shows the basic molecular structure of asphaltenes.

Asphaltenes are usually appeared as a dark brown to a black friable, sticky, semisolid fraction that has no certain melting point, generally foam and swell to leave a carbonaceous residue upon heating. They are formed from petroleum by the inclusion of a nonpolar solvent (hydrocarbon) with a surface tension lesser than that of 25 dyne $\mathrm{cm}^{-1}$ at $25^{\circ} \mathrm{C}$ $\left(77^{\circ} \mathrm{F}\right)$. A study on the solubility of the asphaltenes has been done. Asphaltenes are soluble in a liquid with a surface tension greater than 25 dyne $\mathrm{cm}^{-1}$. However, asphaltenes are insoluble in the most liquefied petroleum gases which are economically needed to be separated upon dealing out

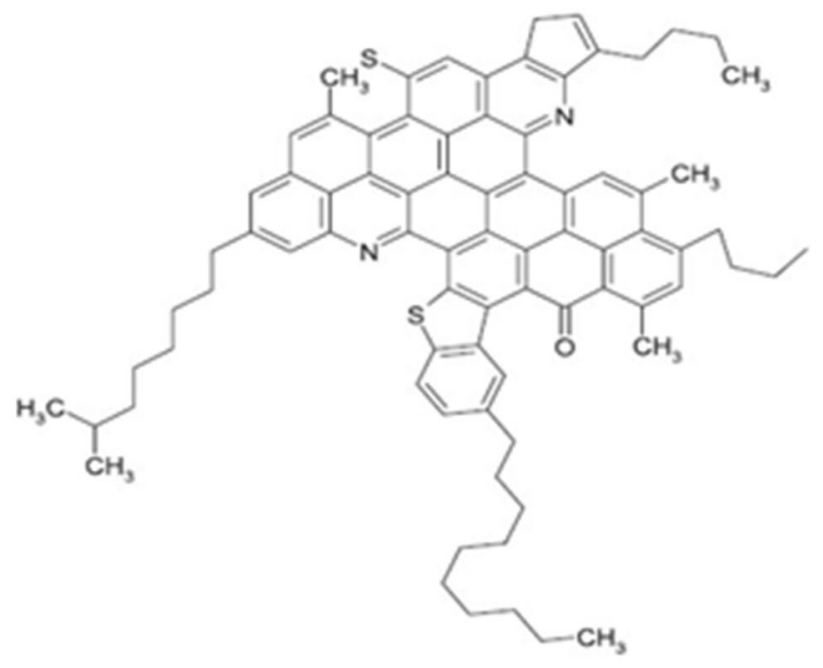

Fig. 7 Molecular structure of asphaltenes suggested by López-Chávez et al. (2007)
مدينة الملك عبدالعزيز

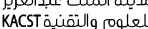
Springer 
petroleum residues deposited for asphaltenes and resins (Speight 1982, 2014).

The existence of asphaltenes is acknowledged to have a critical effect on both processability and recovery of crude oil and to deal with the emulsion stability. Certainly, the presence of asphaltenes promotes low rate separation (precipitation, phase separation) from the oil medium which also resulted from the deposition of solids during recovery operations. Phase separation will cause coke formation during processing as well as the failure of an asphalt pavement by loss of physical structure of the asphalt aggregate system (Speight 1992). Thus, it is significant to understand the structure and stability of crude oil, and the one way in which to improve the understanding is by an investigation of asphaltene precipitation/flocculation, by titration, using solvent/nonsolvent mixtures. Some examples and structures of surfactant molecule are shown in Fig. 8.

Emulsifying agents are responsible for the development of emulsions. Hydrophilic-lipophilic balance (HLB) who first demonstrated by Griffin (1949) is the most adequate way to test the effectiveness of the demulsifier. The two main dissimilar parts: hydrophilic head (water liking) and a hydrophobic tail (water disliking) contain in the amphiphilic molecules like surfactants. The hydrophilic part of the surfactant molecule can be positive (cationic), negative (anionic), neutral (nonionic), or zwitterionic (amphoteric). However, the hydrophobic part is comprised of one or more hydrocarbon chains, commonly with 6-22 carbon atoms (Umar et al. 2018; Migahed and Al-Sabagh 2009). Griffin established that alkoxylated nonionic surfactants are more successful than with ionic surfactants.

A calculation of HLB number from its chemical structure that enables to determine the surfactants to be used in water-in-oil emulsion or oil-in-water emulsion was proposed. By adding some surfactants in water and shaking it, an approximate HLB number can be attained. For example, $\mathrm{HLB}=\%$ of the hydrophilic group (molar) was divided by 5 ; hence, the largest HLB number is 20 and a completely watersoluble surfactant is performed. The lowest HLB number, zero, is favorable to represent a completely water-insoluble

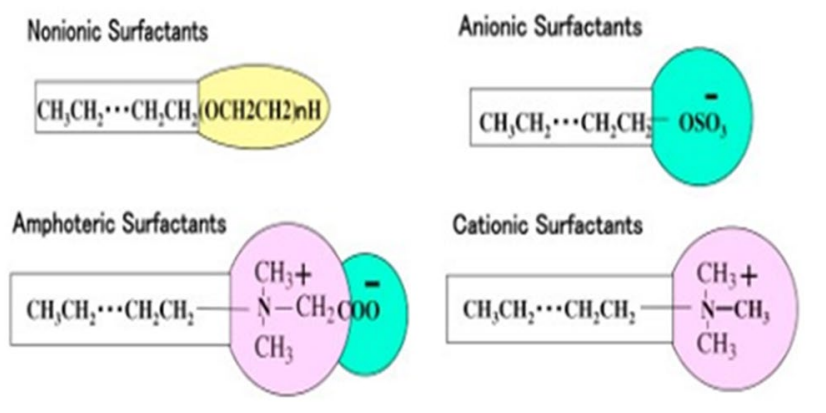

Fig. 8 Examples and structure of surfactant molecule (Nakama 2017) surfactant. The calculation of the HLB number resulted that for water-in-oil emulsion, surfactants with a high HLB number can be used. However, surfactants with a low HLB number can be applied for oil-in-water emulsion (Porter 1991). Table 2 shows the HLB number with an appearance on adding surfactant on water.

Significantly, the emulsions need to be treated by separating the dispersed water from the oil to fulfill the market requirement and transportation provision and to decrease the problems accounted for by the stable emulsion. Furthermore, the well-organized treatment of the emulsion may reduce erosion and catalyst poisoning in downstream operation amenities (Kokal and Aramco 2005). The separation mechanism of the emulsion is indispensable, and many oilfield researchers had proposed various ways to accomplish the targeted treatment.

\section{Oilfield demulsifications}

The difficulties associated with the process of emulsion give a tremendous effect, and it becomes the main issue in the petroleum industry. At present, demulsification approaches have been the major attention among the leading studies. The narrowness of the future needs to overcome the difficulties associated with crude oil emulsions and requires consideration of several factors so that it can be broken easily, such as physical and chemical properties of the crude oil, production temperature, distance between reservoir and topsides, degree of agitation experienced between reservoir and separator and turbulent flow presence of solid that enhances the formation of stabilized emulsion.

Demulsification is a process for the separation of the emulsion into two phases. There are three basic methods for demulsification: physical, chemical and biological, and the effectiveness of these methods can be observed on the ability in reducing the emulsion stability until the two phases separate (Zolfaghari et al. 2016). In the petrochemical industry, the emulsion must be separated into two phases to ensure that refining process goes smoothly (Abdulredha et al. 2018).

Table 2 The appearance of water depends on the HLB number (Porter 1991)

\begin{tabular}{ll}
\hline HLB number & $\begin{array}{l}\text { Appearance of adding } \\
\text { surfactant on water }\end{array}$ \\
\hline $1-4$ & Insoluble \\
$4-7$ & Poor dispersion unstable \\
$7-9$ & Stable opaque dispersion \\
$10-13$ & Hazy solution \\
$13-20$ & Clear solution \\
\hline
\end{tabular}


The main reason for this priority is concerning the problems resulted from the formation of crude oil emulsions. If the demulsification process is not running, many problems will arise as follows:

- Overloading of surface separation equipment

- Pressure growth due to the high viscosity of the emulsion

- Risk of corrosion and catalyst poisoning

- Many basic sediments, water and salts are sent to the refinery

- The high cost of pumping and vessel heating

It is compulsory to study about the characteristics and properties of emulsions and which mechanisms involve during the coalescence of the water droplets to generate high separation rate between the coproduced water and the oil phase in an emulsion (Ese et al. 1999). Apart from that, the study of the suitable chemical additives to treat these emulsions also known as demulsifiers is essential to provide efficient demulsification process (Abdulredha et al. 2018).

\section{Demulsifiers}

Demulsifiers are a group of privilege chemicals or surfactants used to separate water content in the water-in-oil and oil-in-water emulsions usually at low concentrations. During production, the crude oils have a weighty amount of saline water (produce water). This saline water should be separated from the crude to avoid difficulties during the refining process. The major reason is that this saline water may lead to corrosion in the facilities which posed crucial problems to the industry.

In 2018, according to Abdulredha et al. (2018), demulsifiers or surfactants are organic particles comprising of two parts: the polar portion that is attractive to the water phase (hydrophilic) and the nonpolar portion that is attractive to the oil phase (hydrophobic). Commonly, demulsifiers can be categorized into four groups: surfactant nonionic, ionic, amphoteric and polymer surfactant.

The demulsifiers' purpose is to drop the water to a low point in the system where it can periodically be eliminated. They prevent the formation of oil and water mixture. Some demulsifiers are polymers, and others have structures like nonionic emulsifiers. Demulsifiers' chemicals involve desalters and deoilers, which is considered to be about $40 \%$ approximately the world oilfield production chemicals market. There is an escalating interest among the researchers in the demulsifiers' chemicals in breaking the crude oil emulsions system.

\section{Basic structure of a demulsifier}

In general, water-soluble demulsifiers are used to destabilize oil-in-water emulsion and oil-soluble demulsifiers are commonly suitable to destabilize water-in-oil emulsions. This finding is supported by Wong et al. (2015) who invented water-soluble polymer as water-soluble demulsifier but also effective as oil-soluble demulsifier counterpart. They found that oil-soluble demulsifier is risky to handle and use pricy chemicals such as ethylene and propylene oxide. When the demulsifier is added to the crude oil, they discovered the dispersed water droplets and thus break the water-in-oil emulsion. The basic structure of demulsifier is illustrated in Fig. 9.

\section{Chemical demulsifiers}

Due to the presence of the surfactants such as asphaltenes and resins, a chemical additive is required to inhibit the gross formation of the emulsion (Feitosa et al. 2019). A correlation exists in a study about the ability of a demulsifier to break the water-in-oil emulsion that contains unwanted lipophilic surfactants referred to as asphaltenes. In agreement with the physicochemical formulation concept, demulsifier is a hydrophilic surfactant which can obtain optimum formulation at the water and oil interface. As reported in this study, the demulsifier involves the mixture of lipophilic and hydrophilic surfactants which are capable of performing demulsification. It can produce a compromising behavior between the components. Thus, with some synergistic effect, the mixture usually can promote a good performance as a demulsifier (Sjöblom 2001; Delgado-Linares et al. 2016).

Grenoble and Trabelsi (2018) had carried out a study on optimum demulsifier formulations based on hydrophilic-lipophilic balance. They claimed that the main objective of a demulsifier system is to prevent the emulsification mechanism by readily adsorbing at the interfacial phase

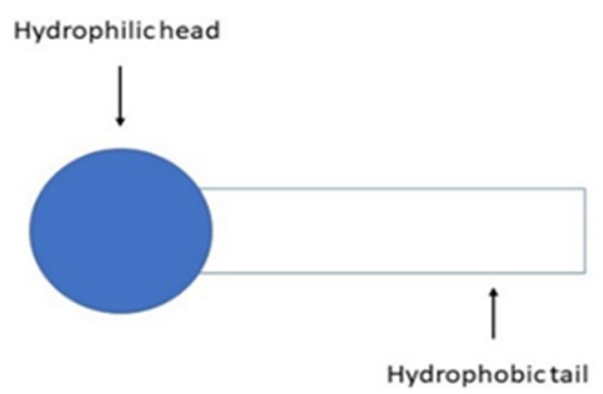

Fig. 9 Basic structure of demulsifier (Porter 1991) 
and weakening the asphaltenes or resins layer associated with the crude oils. To promote a good destabilization at the interfacial phase, there are several mechanisms need to be addressed. As such, the demulsifier must competitively adsorb at the interface, remove and break up the asphaltenic aggregates as well as reduce the interfacial tension between the hydrocarbon and aqueous phases and hence facilitate the droplet coalescence kinetics (Salager and Forgiarini 2012).

\section{Nanoparticle-modified demulsifiers}

Due to the existence of intense acidity in several heavy crude oils, many problems were faced by the refiners, especially corrosion in the wellhead, oil pumps, valves and production facilities, which might also produce low profits of oil companies. An investigation to remove the acidity using nanofluid demulsifier on heavy Iranian crude oil was performed by Pasban et al. (2017). The objective of this study is to eliminate the high acidity concentration to less than $1 \mathrm{mg} \mathrm{KOH} / \mathrm{g}$ of sample. Most of the refineries in the world are designed for the crude oils with a total acid number less than 0.5. They found out that water formation was the main factor for increasing of acid number in the crude oils.

Their studies revealed that temperature plays the most important role to separate the salt and water as well as reduce the acidity of crude oils. As the viscosity of the crude oil decreases, the temperature increases, hence facilitating the separation of the inorganic and aqueous phases. In the experiment, under the optimum condition, a temperature above $100{ }^{\circ} \mathrm{C}$ and centrifugation speed above $2500 \mathrm{rpm}$ showed the best performance in the separation. The result also demonstrated that the acid number of heavy crude oils would reduce to $80 \%$ by eliminating the formation of water of the crudes. The demulsifiers were designated based on API gravity, asphaltene content and molecular weight of the crude oils. The best results were obtained using the nanoparticles' demulsifier selected and are listed in Table 3.

\section{Classification of surfactants}

Surfactants are categorized economically based on their usage, dissociation in water and charge carrier (Salager 2002; Umar et al. 2018; Schramm et al. 2003). Surfactants

Table 3 Physical properties of demulsifier (Pasban et al. 2017)

\begin{tabular}{ll}
\hline Appearance & Viscous liquid \\
\hline Color & Cloudy \\
Odor & Not detected \\
Flashpoint, ${ }^{\circ} \mathrm{C}$ & 120 \\
$\mathrm{pH}$ & $7-9$ \\
Density @ $25^{\circ} \mathrm{C}, \mathrm{g} / \mathrm{cm}^{3}$ & 1.049 \\
\hline
\end{tabular}

that are dissociated in water into an amphiphilic anion and a cation, usually alkaline metal $\left(\mathrm{Na}^{+}, \mathrm{K}^{+}\right)$or quaternary ammonium, are known as anionic surfactants. They are comprehensively used in most industries and conquer about half of the surfactant world production. They are composed of alkylbenzene sulfonates (detergents), (fatty acid) soaps, lauryl sulfate (foaming agent), di-alkyl sulfosuccinate (wetting agent) and lignosulfonates (dispersants). The charge carrier is negative, as in soap: $\mathrm{C}_{17} \mathrm{H}_{35} \mathrm{COO}^{-} \mathrm{Na}^{+}$, sodium stearate. Apart from that, cationic surfactants are dissociated in water into an amphiphilic cation and an anion, most frequently of the halogen type. The high-pressure hydrogenation reaction needs to be achieved during their synthesis causing these surfactants to be more costly if compared to anionic surfactants. As a result, they are only applied in situations where there is no cheaper alternative. They hold positive charges, as in quaternary ammonium salts: $\left(\mathrm{C}_{18} \mathrm{H}_{37}\right)_{2} \mathrm{~N}^{+}\left(\mathrm{CH}_{3}\right)_{2} \mathrm{Cl}^{-}$, dimethyl dioctadecyl ammonium chloride. Besides, when a single surfactant molecule displays both anionic and cationic dissociations, the surfactant is recognized as an amphoteric or a zwitterionic surfactant. For instance, synthetic products are such as betaines or sulfobetaine and natural substances like amino acids and phospholipids. In the molecule, positive and negative charges exist to provide solubility as in $\mathrm{C}_{12} \mathrm{H}_{35} \mathrm{~N}^{+}\left(\mathrm{CH}_{3}\right)_{2} \mathrm{CH}_{2} \mathrm{CH}_{2} \mathrm{COO}^{-}$, B-N-alkyl amino propionic acid. Besides, nonionic surfactants increased by about $45 \%$ of the total production worldwide. The hydrophilic group contains a nondissociating behavior; thus, these surfactants do not ionize in the aqueous solution. As a result, they are suitable with other complex mixtures as in many commercial products. The presence of solvation of polyoxyethylene oxide groups provides solubility as in $\mathrm{C}_{9} \mathrm{H}_{19} \mathrm{C}_{6} \mathrm{H}_{4}\left(\mathrm{OCH}_{2} \mathrm{CH}_{2}\right)_{9} \mathrm{OH}$, nonylphenol ethoxylate. The most formed demulsifiers are ionic and nonionic demulsifiers. According to Matijasevic and Banhart (2006), the classification of ionic demulsifiers is oil-soluble- and water-soluble-type demulsifiers, and their properties are shown in Table 4.

\section{A general theory of demulsifiers action}

In Handbook of Emulsion Technology (Sjöblom 2001), the correlation of pure solvents and low molecular weight (LMW) and high molecular weight (HMW) demulsifiers can demonstrate demulsification performance. Low molecular weight demulsifiers' functionality is based on two distinct mechanisms: increased interfacial activity and change in the wettability of stabilizing components. However, for the high molecular weight demulsifiers to operate optimally, time is required for the diffusion at the interfacial membrane. The two categories of demulsifiers mentioned are represented by the chemicals added. They disclosed that in addition to the LMW chemical additives, the average age separation

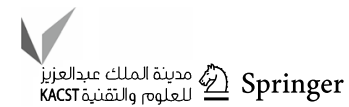


Table 4 Comparison between oil-soluble and water-soluble demulsifiers (Matijasevic and Banhart 2006)

\begin{tabular}{ll}
\hline Oil-soluble demulsifiers & Water-soluble demulsifiers \\
\hline 2000-50,000 molecular weight (mw). Large mw is preferred & $10,000-15,000$ molecular weight (mw). Low mw is preferred \\
Polydisperse interfacial active polymers & Tetra polymer or penta polymer \\
Tetra polymer or penta polymer & The polymer presents hydrophilic (-COOH) and hydrophobic (alkyl) groups. \\
Risky and costly chemicals (ethylene, propylene oxide) & Easy-to-handle chemicals (methyl methacrylate, butyl acrylate, acrylic acid, \\
& methacrylic acid) \\
\hline
\end{tabular}

without any additional chemicals is $21.5 \%$ of water after $30 \mathrm{~min}$, which reduce the separation. Apart from that, the addition of HMW chemical additives shows a better water separation. These results are related to interfacial films and added species.

\section{Demulsification mechanisms}

To date, many researchers (Yang et al. 2018; Adewunmi and Kamal 2019; Biniaz et al. 2016) had demonstrated the demulsification mechanisms of water-in-crude-oil emulsion as well as of crude-oil-in-water emulsions (Martínez-Palou et al. 2013; Tao et al. 2015). The studies of both types of emulsions are in mutual understanding of the emulsion stability formation and future needs to break the emulsion.

To efficiently overcome the limitations discharged by the occurrence of stable emulsions, the chemical, membrane and electrostatic demulsifications are the most prevalent techniques (Nikkhah et al. 2015; Behroozi et al. 2019). Destabilization of the crude oil emulsions is enhanced by increased temperature, centrifugation, electrical methods, high resonance time and chemical treatment separation. Numerous approaches were found for demulsification, and many parameters also are taken into consideration to achieve it, such as droplet size distribution, dosage, and drainage rate, the viscosity of the emulsion, temperature and type of emulsifier/demulsifier (Zolfaghari et al. 2016).

\section{Chemical demulsification}

Emulsification and demulsification both are intricate operations, and in a simple understanding, demulsification is the opposite of the emulsification process. Chemical demulsification has long been known and applied in the petroleum industry because the mechanism is the cheapest, most convenient and efficient in the separation of water-in-oil emulsions (Matijasevic and Banhart 2006).

The interaction of three major effects during the investigation of kinetic chemical demulsification operation is (Ese et al. 1999):
- The displacement of the asphaltenic film from the water/ oil interface by the demulsifier

- Flocculation or aggregation

- Coalescence of water droplets

The overview processes of the emulsion treatment are captured in Fig. 10 (Auflem 2002). The emulsions undergo creaming, flocculation or sedimentation before coalescence process takes place. This finding was also agreed by Kokal and Aramco (2005) where flocculation or aggregation process always takes first before the coalescence process. They hold that flocculation of water droplets could make muddy water turning into clear water by adding some flocculants. The droplets tend to accumulate by forming aggregates, and the position of the droplets is adjacent to each other and may come in contact at some point. At the end of the process, colloids formed in the muddy water enable the water droplets that clumped together to form flocs or aggregates. Numerous aspects such as water cut, temperature, the viscosity of the oil and the density difference between the oil and water may affect the rate of flocculation.

Coalescence is an irreversible process that follows the flocculation of the water droplets. During coalescence, the small dispersed water droplets tend to accumulate and form a bigger droplet, thus reducing the number of water droplets.

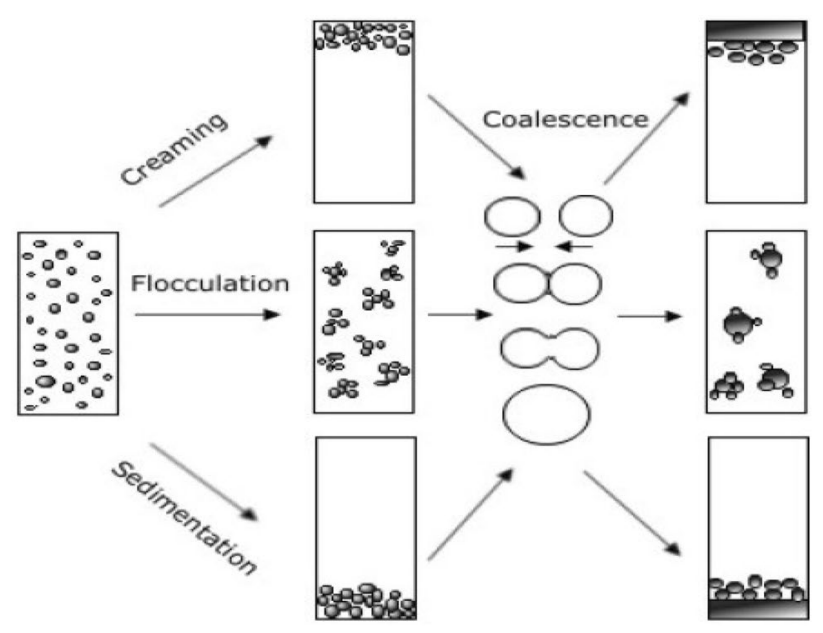

Fig. 10 Processes of emulsions treatment (Auflem 2002) 
When the droplets combined, the thin film in the continuous phase will be trapped between the droplets; thus, the stability of the emulsion can be determined (Sjöblom 2001). Several factors can facilitate the coalescence process, such as high rate of flocculation, absence of mechanically strong films, high IFTs, low oil and two phase viscosities, low concentration of surfactants at the interphase, high temperature and water cuts (Kokal and Aramco 2005).

\section{Performance demands on demulsifiers}

Once the demulsification process is carried out, the main thing to focus on is the basic demands on the demulsifier performance and its ability to have the following behaviors: (1) strong appeal to oil/water interface with the capability to destabilize the film surrounds the droplets and/or to vary the contact angle of the solids part of the interfacial film; (2) capability to flocculate droplets; (3) capability to develop coalescence by welcoming pathways to water's natural attraction to water; and (4) offering inhibition of film drainage and thinning by inducing changes to the interfacial rheology (Sjöblom 2001). The demulsifier may affect the droplet interfacial film by displacement and complexation, varying the solubility in the continuous phase and varying the viscosity of the interfacial film or adsorption which promotes film drainage.

\section{Chemical demulsifier as emulsion treatment}

Nikkhah et al. (2015) proposed novel nano-titania as a modified chemical demulsifier for the water-in-oil emulsion. In this study, a sol-gel method was performed as an alternative to synthesis the nano-titania particles. Then, the research continues to analyze the nano-titania particles by particle size analysis (PSA), X-ray diffraction (XRD), transmission electron microscopy (TEM), bottle test, electrostatic test and standard IP77 method. The results show that the sample with the greatest water separation employed by the nanotechnology can promote $90 \%$ demulsification efficiency besides reducing the time taken for the water separation.

In 2016-2019, as reported by Biniaz et al. (2016), Hazrati et al. (2018), Adewunmi and Kamal (2019), the water-in-oil emulsion can be separated using ionic liquids. In the study conducted by Hazrati et al. (2018), they presented imidazolium ionic liquids with long alkyl chains which coupled with $\mathrm{Cl}$, PF6 and NTf2 anions as an active demulsifier. The experiment was carried out by observing the interfacial tension between the water and crude oil, and within 1-, 2-, 6and 24-h interval, a bottle test was performed. Three factors were considered: the alky chain length of cation, anion type and IL dosage in three levels to obtain a good behavior of demulsifier. As a result, they attained $86-95 \%$ water separation efficiency and $0.7-6.26 \mathrm{mN} / \mathrm{m}$ for interfacial tension
(IFT). They concluded that the longer the alkyl chain length of the ionic liquids, the higher the demulsification efficiency as well as IFT reduction.

Feitosa et al. (2019) had investigated additives based on cardanol to study the demulsification mechanisms. The crude oils used were three Brazilian crude oil with $30 \%$ (v/v) brine cut, 60 and $240 \mathrm{~g} / \mathrm{L} \mathrm{NaCl}$ of salinity, at $\mathrm{pH}$ 3 to 10 in a constant agitation (3200 rpm). In this work, four types of chemical additives (hydrogenation, ethoxylation, formaldehyde polycondensation and ethoxylation of formaldehyde polycondensation) were approached to synthesize new products from cardanol. FTIR and ${ }^{1} \mathrm{H}$ NMR analyses were done to characterize the additives. Using bottle tests, the experiments were performed at temperature $60{ }^{\circ} \mathrm{C}$, and additional $200 \mathrm{ppm}$ of the chemical was tested. The results showed that ethoxylated compounds, at neutral $\mathrm{pH}$, are more suitable to separate water-in-oil emulsions.

In 2018 , the dosage and stability analyses were examined, whereas in this work, a novel tannic acid phenol amine (TAPA) polyether as the demulsifier of water-inaging-crude-oil (WACO) emulsion was examined ( $\mathrm{Li}$ et al. 2018). Li had found out that this framework enhanced the demulsification effectiveness by $91.7 \%$ within a period of $90 \mathrm{~min}$ of laboratory test and on the offshore platform can achieve $97.9 \%$ efficiency within $45 \mathrm{~min}$. Many parameters are considered such as measurement of hydrophilic-lipophilic balance (HLB), interfacial task, multiple aromatic rings and relative solubility number (RSN); however, the changes in the water droplets are the main interest in the

Table 5 Demulsifiers and their types (Fink 2012)

\begin{tabular}{ll}
\hline Demulsifier & $\begin{array}{l}\text { Type of } \\
\text { demulsi- } \\
\text { fier }\end{array}$ \\
\hline Tannin-based blends & $\mathrm{W} / \mathrm{O}$ \\
Ethoxylated or epoxidized PAG & $\mathrm{W} / \mathrm{O}$ \\
Oxalkylated poly(alkylene) poly(amine)s & $\mathrm{W} / \mathrm{O}$ \\
Polythioalkyloxides & $\mathrm{W} / \mathrm{O}$ \\
Poly(ether) poly(urethane) & $\mathrm{W} / \mathrm{O}$ \\
Diallyl dimethyl ammonium chloride polymer & $\mathrm{O} / \mathrm{W}$ \\
Amphoteric acrylic acid (AA) copolymer & $\mathrm{O} / \mathrm{W}$ \\
Copolymer of polyglycol acrylate & $\mathrm{O} / \mathrm{W}$ \\
Poly(1-acryloyl-4-methyl piperazine & $\mathrm{O} / \mathrm{W}$ \\
Vinyl phenol polymers & $\mathrm{O} / \mathrm{W}$ \\
Poly(dimethyl diallyl ammonium chloride) & $\mathrm{O} / \mathrm{W}$ \\
Cross-linked oxalkylated poly(alkylene) poly(amine)s & $\mathrm{O} / \mathrm{W}$ \\
Dithiocarbamates & $\mathrm{O} / \mathrm{W}$ \\
Sulfonated poly(styrene)s & $\mathrm{O} / \mathrm{W}$ \\
Acid-modified polyol & $\mathrm{LS}$ \\
\hline
\end{tabular}

$L S$ liquid-solid separation, $O / W$ oil-in-water use, $W / O$ water-in-oil use 
discussion. They concluded that TAPA can act as an efficient demulsifier for WACO. Common chemical demulsifiers are listed in Table 5.

\section{Nuclear magnetic resonance (NMR) perspective}

Fridjonsson et al. (2014) had presented a procedure that implements nuclear magnetic resonance (NMR) to break the emulsion. As a result, the optimized droplet sizing of water-in-crude-oil emulsions is well demonstrated. They came up against the difficulty where the water droplet size distribution is incorrectly provided by the NMR signal from the oil phase. However, to overcome the inaccurate crude oil distribution, they discovered an optimization routine utilizing a definite chemical-shift selection of water droplet rendered by high-field superconducting spectrometers. The results showed that numerous NMR measurement timing parameters could be adjusted and greatly optimize the droplet sizing of water-in-oil emulsions.

\section{Demulsifier inhibition of film creating and coalesce droplets}

Yang et al. (2018) had performed a study on demulsification mechanisms using a modified thin liquid film (TLF) technique. In the experimental works, an altered design of Scheludko-Exerowa cell with a dosing mechanism is well demonstrated. An inclusion of biodegradable polymer demulsifier (EC300) is allowed on the intervening oil film, and by observing the thinning process of the intervening oil film after the addition of the demulsifier, the formation of the stabilized water-in-oil emulsion and the demulsification techniques is well understood. The results showed that an unstable film oil is formed with a lifetime of below $20 \mathrm{~s}$ from the original stable film formed by asphaltene-inheptol solutions. The changes in the stability and drainage rate acknowledged the overdosing EC300 demulsifier can distinguish the asphaltene formation at the oil-water interphase using a modified TLF technique.

In 2018, another technique using demulsifier and electrocoalescence in water-in-crude-oil emulsions that supported film thinning under DC electric fields was reported (Mhatre et al. 2018). They studied the effect of asphaltene flocculation and various chemical demulsifiers to practice the electrocoalescence. In the experiment, to exhibit the progress of the coalescence, a linear rising DC electric field to the thin layer of emulsion and current flowing through the emulsion with the usage of parallel plate electrode cell was observed. At the first phase, they found out that the asphaltene flocculation gave a tremendous effect to the electrocoalescence process. In the next phase, they disclosed that demulsifiers contain concentration limit because raising their concentration does not enhance the electrocoalescence process. The rate of separation is higher when adding demulsifier before the dispersion of water than adding demulsifier after in electrocoalescence. A model for qualitative prediction of film drainage between two demulsifiers laden with water-in-oil interfaces also is presented.

In 2019, an ultrasonic standing wave coalescence chamber was approached by Atehortúa et al. (2019) to separate water-in-oil emulsions. In the coalescence process, the standing wave coalescence chamber which consists of highfrequency ultrasonic enables to lower the dosage of chemical demulsifiers. The experiment performed in the laboratory processing plant and experimental conditions such as temperature, flow rate, initial water content and amount of demulsifier is considered in the ultrasound application. They analyzed that acoustic radiation force can facilitate demulsification better than gravitational separation technique.

\section{Graphene oxide as a demulsifier medium}

Graphene becomes the large interest because of its cheap cost, easy to find and the broad capabilities to convert (Alam et al. 2017). Graphene oxide is a two-dimensional
Fig. 11 Structure of a a single graphene sheet and $\mathbf{b}$ graphene oxide (GO) (Alam et al. 2017)

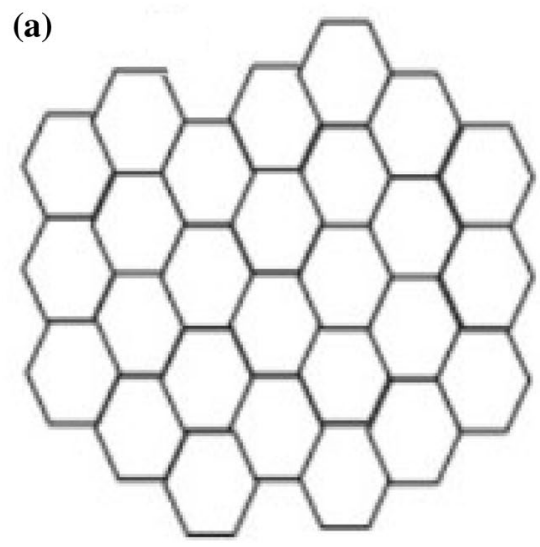

(b)

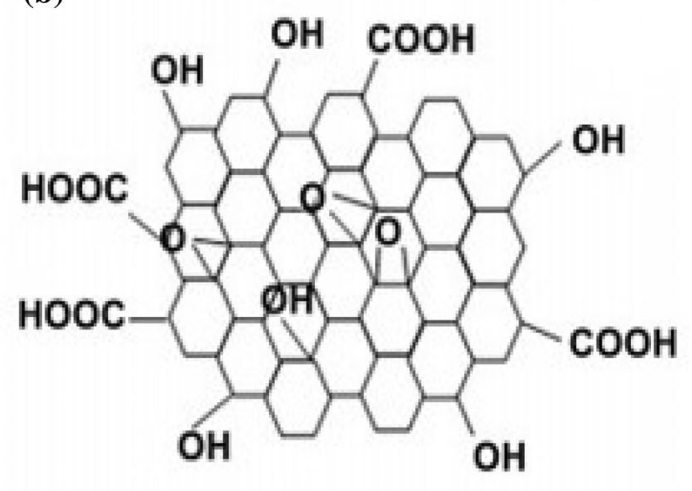


(2D) honeycomb lattice, comprising of a flat monolayer of carbon atoms arranged in a hexagonal lattice structure, and is a basic layer of graphitic materials which are the building block (Geim and Novoselov 2017). Figure 11 shows the structure of graphene oxide.

From 2015 to 2018, an efficient and rapid demulsification of crude-oil-in-water emulsions driven by graphene oxide (GO) has agreed by Liu et al. (2015, 2017), Fang et al. (2016) and Contreras Ortiz et al. (2018). At an optimum GO dosage, $99.9 \%$ of demulsification efficiency was discovered (Liu et al. 2015). The $\mathrm{pH}$ value of the emulsion also plays an important role to determine the distribution of GO either in oil or water phase after demulsification. The rapid demulsification was enhanced by the strong adsorption between GO nanosheets and asphaltenes films supported by $\pi-\pi$ and/or $n-\pi$ interactions. This finding is maintained by Liu et al. (2017) who claimed the $\pi-\pi$ and/or $n-\pi$ interactions between the GO materials and asphaltenes molecule are the primary factor that facilitates the demulsification. They successfully presented recyclable magnetic graphene oxide to separate the oil and water interfaces. According to Fang et al. (2016), they found out that the presence of GO decreases the interfacial tension of the emulsion. In the experiment, the interfacial rheology properties and interfacial tension were studied accordingly. Also, Contreras Ortiz et al. (2018) tested the separation of crude-oil-in-water emulsions using GO and amine-modified GO particles. An amphiphilic material, which is graphene oxide functionalized by amino groups, was prepared by Hummer's modified method, Fourier transform infrared spectroscopy (FTIR), Raman spectroscopy, X-ray diffraction (XRD) and emission scanning electron microscope (SEM), which were used to characterize it. They disclosed that less time is required to break up the crude-oil-in-water emulsions.

\section{Properties of demulsifier as an advantage}

Li et al. (2017) had presented nanoporous divinylbenzene (DVB) $/ \mathrm{SiO}_{2}$ hybrid material as an effective separation of surfactant-stabilized water-in-oil and oil-in-water emulsions. They reported that the hybrid material had superiority that facilitates the separation. As such, it has a large specific surface area and nanopore size distribution and was associated with its superhydrophobicity. Furthermore, the hybrid material with multi-level nanopores accompanied by hierarchical nanoporous structure enhances the absorption capacity of the organic solvents from the selective water and provides a good reusability, meaning that it remains after the separation process occurring again. In conclusion, they greatly proved that the hybrid material characteristic can illustrate a potential separation of both water-in-oil and oil-in-water emulsions.
All in all, numerous studies on the demulsification were engrossed in the chemical demulsification, where dewatering the emulsions with aided chemical additives and displacing the droplet interfacial film lead to film drainage and thus facilitate to break up the emulsions. It is significant to remark the formation of the emulsion, which emulsions are formed, and appropriate approaches are needed to treat such emulsions. The study of the demulsification prevents regular complications occurring in the crude oil flow transport. Several current demulsification techniques are listed in Table 6 .

\section{Demulsification formulation}

The characterization of the emulsion stability and demulsification performance depends on several properties such as basic sediment and water, droplet size distribution, bulk, interfacial viscosities and conductivities (Sjöblom 2001; Kokal and Aramco 2005). The parameters involved are deliberated below:

\section{Droplet size distribution}

The droplet size and distribution of the oilfield emulsions both influence the demulsification performance. The droplet diameter and drop size of both water-in-oil emulsion and the oil-in-water emulsion is greater than $0.1 \mu \mathrm{m}$ and $100 \mu \mathrm{m}$, respectively, which are relatively small. According to Zolfaghari et al. (2016), the small droplet size and distribution eventually lead to high viscosity and concentrated emulsions. Subsequently, it will take longer time to coalesce the dispersed drops and finally sediment (water globules) or float (oil droplets). When the average droplet size decreases, it will indicate longer residence time and thus decrease the rate of separation. An effective demulsifier is required to aid the coalescence process, thus promoting a decent demulsification performance (Fridjonsson et al. 2014).

\section{Temperature}

Heat plays an important role and provides both advantages and disadvantages to the destabilization of emulsions. Increment of temperature influenced the viscosity and density differences of the water and the crude oil, and then, they begin to diverge. Besides, it produces a high number of droplets collision and low oil viscosity, which will reduce the strength of the accompanied stabilizing agents at the droplets and thus provide higher chances of settling (Mohammed et al. 1994). Consequently, a lower interfacial shear viscosity of crude oil will improve the diffusion rate of film drainage between adjacent drops. In some other cases, high temperature will degrade the performance of demulsifier as the chemical additives are sensitive to heat (Sjöblom 2001).

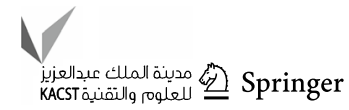




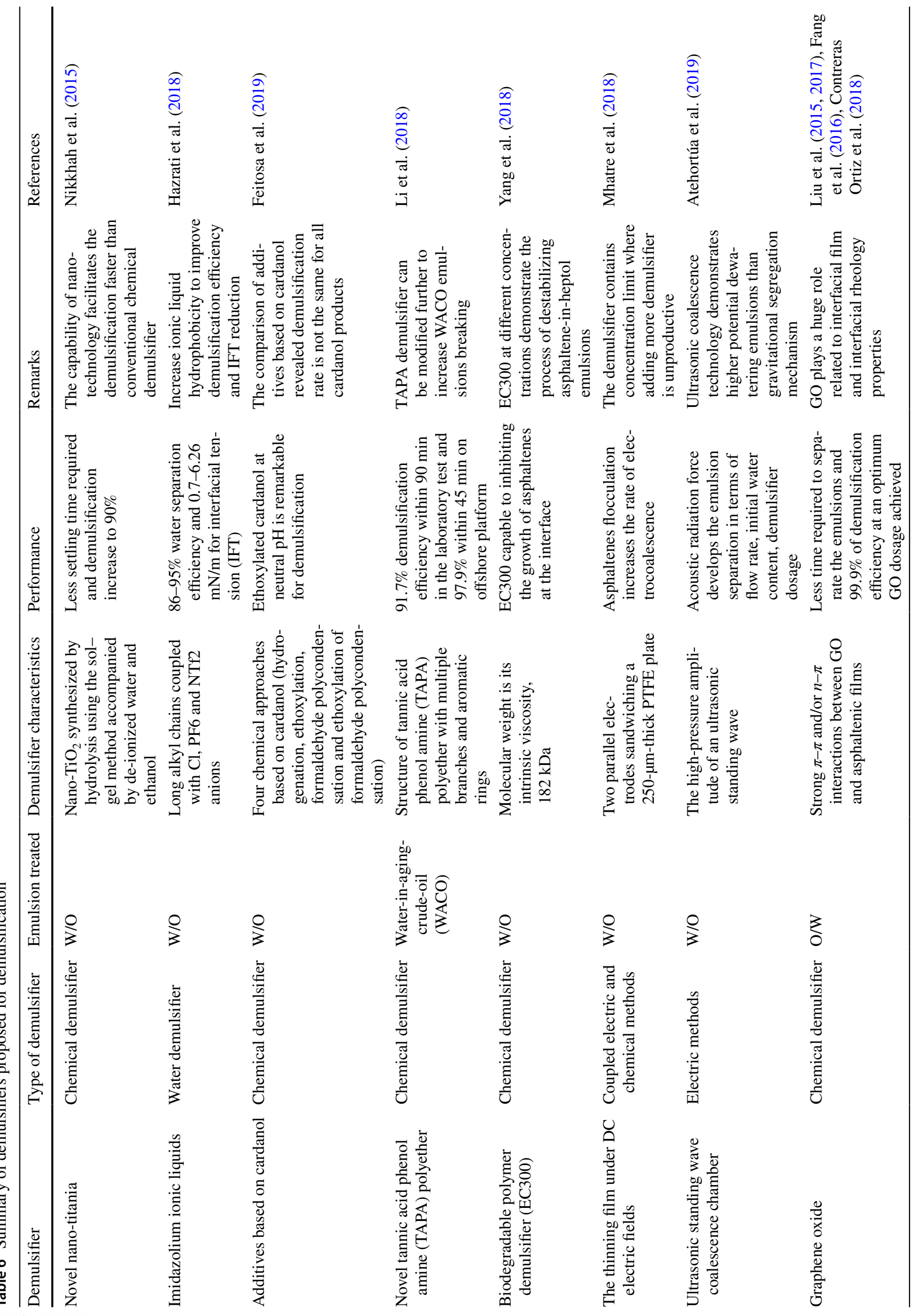


Generally, Zolfaghari et al. (2016) stated that in emulsion stability, an oil phase and brine compositions in the emulsion are two primary factors that affect the water $\mathrm{pH}$. The higher the $\mathrm{pH}$ value of emulsion, the higher the hydrophilicity of surfactants. In line with this finding, W/O emulsions are reliable to produce in low $\mathrm{pH}$ surroundings and $\mathrm{O} / \mathrm{W}$ emulsions are generated in high $\mathrm{pH}$ environment. In a study by Fortuny et al. (2007) by addition of $\mathrm{NaOH}$ concentration in the system, they compared the demulsification efficiency between emulsions with $\mathrm{pH}$ value of 7.0 and 9.5. As a result, they verified that a slight increase in demulsification efficiency falls to the emulsion at $\mathrm{pH}$ 7.0. Consistent with the finding, the growth of emulsion $\mathrm{pH}$ will weaken the stable emulsion because asphaltene films are reliably developed in the acidic medium before the emulsion becomes unstable in alkaline surroundings. At optimal $\mathrm{pH}$ where the rate of separation is maximum, the demulsifier used is recognized as amphoteric surfactants, in oil-brine systems (Zolfaghari et al. 2016).

\section{Water content and oil content}

Besides asphaltenes and resins, water content and oil content in water-in-oil emulsions are also the predominant parameters influencing the emulsion separation efficiency and the stability of emulsion. Principally, the presence of demulsifier can promote the demulsification efficiency with increasing water content in the emulsion, along with decreasing time and concentration of demulsifier doses required for water separation, provided that the percentage of water content is in range from 0.3 to 0.7 volumetric fraction (Zolfaghari et al. 2016; Abdulredha et al. 2018). Emulsions with a higher water content are easily broken than the lower content, besides increasing the water content causing the crude oil emulsions to be more viscous (Mouraille and Skodvin 1998). Furthermore, increase in volume of dispersed phase promotes demulsification efficiency and rate of coalescence due to increase in entropy for efficient collision between the single-phase droplets. However, in contrast to the absence of demulsifier, lowering the oil content from 90 to $60 \%$ in a stabilized oil-in-water emulsion causes a little water separation, while reducing the oil content to $50 \%$ will promote the emulsion breaking promptly. An increase in the oil content displays an extremely stable emulsion due to excessive amount of oil in the continuous phase, which may lead in changing from the oil-in-water emulsion to water-in-oil emulsion (Abdulredha et al. 2018). According to Zaki (1997), the oil content on its pour point is important to prevent freezing of mixture in pipelines at low temperatures. This is essential when oil-in-water emulsion is formed to facilitate the transportation of the crude oils by lowering their viscosity.

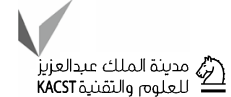




\section{Surfactant molecular weight}

Generally, Grenoble and Trabelsi (2018) stated that molecular weight of a demulsifier renders its mobility and diffusion behavior and at the same time displays interfacial adsorption kinetics. It is observed that demulsifiers with high molecular weights demonstrate low adsorption kinetics, which might demote them as less effective demulsifiers. This is perhaps because the demulsifiers require to cope up with surfactants that exist naturally in crude oil. Ordinally, the adsorption kinetics measured for asphaltenes in crude oils is quite slow (>2-4 wt\%), but it still needs a demulsifier. A high molecular weight demulsifier renders adsorption at the interface and interacts with other particles at the interface, but with moderate adsorption kinetics (Peña et al. 2005).

\section{Agitation velocity and period}

In 2014, R. S.-S. et al. observed the viscosity and droplet size of oil-in-water emulsions which influenced by the speed mixing of the emulsion. In the experiment, they measured the viscosity in a temperature range of $25-65{ }^{\circ} \mathrm{C}$ and the shear stress in the range of 30-200 rpm. Significantly, the result showed that there is a decrease in the droplet size when the mixing speed is risen from 1000 to $1500 \mathrm{rpm}$ and that when we further increase the mixing speed from 1500 to $2500 \mathrm{rpm}$, the droplet size is unchanged. They also found out, as the mixing speed increases from 1000 to $1500 \mathrm{rpm}$, the viscosity of emulsion increases about $150 \mathrm{cP}$. Then, when we further increased from 1500 to $2500 \mathrm{rpm}$, the viscosity only increases about $110 \mathrm{cP}$ (Peralta-Martínez et al. 2014).

The stability of emulsion can be improved by increasing the period and speed mixing of oil-in-water emulsions by decreasing the diameter size of dispersed oil droplets and rise of both emulsion stability and viscosity. This will cause a larger interfacial area that promotes improved particle interaction between the phases. The colloidal surface and Brownian movement forces are proven more powerful than the hydrodynamic forces in systems consisting of dispersed droplets with diameter less than $1 \mu \mathrm{m}$ (Zolfaghari et al. 2016).

\section{Conclusions}

In conclusion, this literature review comprehensively strives for the development of the crude oil emulsions and demulsification process in the petroleum industry. The formation of the emulsions from different platforms has posed many difficulties to the refiners. Out of all demulsification techniques listed, the major purpose is to speed up the interfacial film thinning process by reducing the strength of the stabilizing film as well as impeding the adsorption of emulsifiers at the oil-water interface. An adequate understanding and detailed research into not just the methods to break the emulsions and also the fit-to-purpose demulsifier(s) that can enhance the separation is required to solve the issue of emulsions. These two aspects are significant and have far-reaching implications for the validity of the results described in this review. Besides, the authors noticed that the processes and experimental setup involved in the demulsification are almost constant for all investigations conducted. However, the mechanisms are different from one research to another as we must consider some factors include types and properties of emulsions, roles of solids stabilized emulsions involved and characteristics of demulsifiers that correspond to treat such emulsions. From this review, the concept of the study of demulsification mechanisms by most researchers that involve in the usage of chemical additives that prompt the film drainage is best practiced. Somehow, there are also advantages and disadvantages that can be seen through the demulsification performance. The formulation of the demulsification system was examined to determine the suitable demulsifiers for a certain crude oil. To the best of authors' knowledge, there is slight research on the combination modes of separation and mechanistic modeling of demulsification targeting a higher positive impact on the industry. Therefore, future works would attempt to involve a system typically consisting of a tangible physical aspect and real, solid visible by examining the workings of its individual parts. It is believed that understanding the behavior of emulsions is important to develop a more strategic emulsion-breaking route, as well as impose less economic problems in the petroleum industry. Clearly, the research objectives have been met with the completion of this study in which improvements can be made in the future.

Acknowledgements The author gratefully acknowledges FRGS fund 015 MAO-029 and Universiti Teknologi PETRONAS (UTP), Malaysia, for the financial support. The author also thanks Assoc. Prof Dr. Ismail Mohd Saaid for his help and contribution.

Open Access This article is licensed under a Creative Commons Attribution 4.0 International License, which permits use, sharing, adaptation, distribution and reproduction in any medium or format, as long as you give appropriate credit to the original author(s) and the source, provide a link to the Creative Commons licence, and indicate if changes were made. The images or other third party material in this article are included in the article's Creative Commons licence, unless indicated otherwise in a credit line to the material. If material is not included in the article's Creative Commons licence and your intended use is not permitted by statutory regulation or exceeds the permitted use, you will need to obtain permission directly from the copyright holder. To view a copy of this licence, visit http://creativecommons.org/licenses/by/4.0/. 


\section{References}

Abdulredha MM, Siti Aslina H, Luqman CA (2018) Overview on petroleum emulsions, formation, influence and demulsification treatment techniques. Arab J Chem 13:1-26

Adewunmi AA, Kamal MS (2019) Demulsification of water-in-oil emulsions using ionic liquids: effects of counterion and water type. J Mol Liq 279:411-419. https://doi.org/10.1016/j.molli q.2019.02.008

Alam SN, Sharma N, Kumar L (2017) Synthesis of graphene oxide (GO) by modified hummers method and its thermal reduction to obtain reduced graphene oxide (rGO)*. Graphene 06(01):1-18. https://doi.org/10.4236/graphene.2017.61001

Atehortúa CMG, Pérez N, Andrade MAB, Pereira LOV, Adamowski JC (2019) Water-in-oil emulsions separation using an ultrasonic standing wave coalescence chamber. Ultrason Sonochem 57:5761. https://doi.org/10.1016/j.ultsonch.2019.04.043

Auflem IH (2002) Influence of asphaltene aggregation and pressure on crude oil emulsion stability by Department of Chemical Engineering. Norwegian University of Science and Technology. Doktor Ingeniør Thesis, June, p 58

Behroozi AH, Kasiri N, Mohammadi T (2019) Multi-phenomenal macroscopic investigation of cross-flow membrane flux in microfiltration of oil-in-water emulsion, experimental \& computational. J Water Process Engineering 32:100962. https://doi.org/10.1016/j. jwpe.2019.100962

Biniaz P, Farsi M, Rahimpour MR (2016) Demulsification of water in oil emulsion using ionic liquids: statistical modeling and optimization. Fuel 184:325-333. https://doi.org/10.1016/j. fuel.2016.06.093

Bobra M (1991) Water-in-oil emulsification: a physicochemical study. Int Oil Spill Conf Proc 1991:483-488

Contreras Ortiz SN, Cabanzo R, Mejía-Ospino E (2018) Crude oil/ water emulsion separation using graphene oxide and amine-modified graphene oxide particles. Fuel 240(December 2018):162-168. https://doi.org/10.1016/j.fuel.2018.11.151

Czarnecki J (2009) Stabilization of water in crude oil emulsions. Part 2. Energy Fuels 23(3):1253-1257. https://doi.org/10.1021/ef800 $607 \mathrm{u}$

Czarnecki J, Moran K (2005) On the stabilization mechanism of waterin-oil emulsions in petroleum systems. Energy Fuels 19(5):2074 2079. https://doi.org/10.1021/ef0501400

Davies GA, Nilsen FP, Gramme PE (1996) The formation of stable dispersions of crude oil and produced water: the influence of oil type, wax \& asphaltene content. SPE Ann Tech Conf Exhib. https ://doi.org/10.2118/36587-MS

Delgado-Linares JG, Pereira JC, Rondon M, Bullon J, Salager JL (2016) Breaking of water-in-crude oil emulsions. 6. Estimating the demulsifier performance at optimum formulation from both the required dose and the attained instability. Energy Fuels 30(March):5483-5491

Ese MH, Galet L, Clausse D, Sjöblom J (1999) Properties of Langmuir surface and interfacial films built up by asphaltenes and resins: influence of chemical demulsifiers. J Colloid Interface Sci 220(2):293-301. https://doi.org/10.1006/jcis.1999.6549

Fang S, Chen T, Wang R, Xiong Y, Chen B, Duan M (2016) Assembly of graphene oxide at the crude oil/water interface: a new approach to efficient demulsification. Energy Fuels 30(4):3355-3364. https ://doi.org/10.1021/acs.energyfuels.6b00195

Feitosa FX, Alves RS, de Sant'Ana HB (2019) Synthesis and application of additives based on cardanol as demulsifier for water-in-oil emulsions. Fuel 245(February):21-28. https://doi.org/10.1016/j. fuel.2019.02.081
Fingas M (1995) Water-in-oil emulsion formation: a review of physics and mathematical modelling. Spill Sci Technol Bull 2(1):55-59. https://doi.org/10.1016/1353-2561(95)94483-Z

Fingas M, Fieldhouse B (2004) Formation of water-in-oil emulsions and application to oil spill modelling. J Hazard Mater 107(12):37-50. https://doi.org/10.1016/j.jhazmat.2003.11.008

Fingas M, Fieldhouse B (2009) Studies on crude oil and petroleum product emulsions: water resolution and rheology. Colloids Surf A: Physicochem Eng Asp 333(1-3):67-81. https://doi. org/10.1016/j.colsurfa.2008.09.029

Fink JK (2012) Petroleum engineer's guide to oil field chemicals and fluids. Elsevier, Amsterdam

Fortuny M, Oliveira CBZ, Melo RLFV, Nele M, Coutinho RCC, Santos $\mathrm{AF}$ (2007) Effect of salinity, temperature, water content, and $\mathrm{pH}$ on the microwave demulsification of crude oil emulsions. Energy Fuels 21(3):1358-1364. https://doi.org/10.1021/ef0603885

Fridjonsson EO, Graham BF, Akhfash M, May EF, Johns ML (2014) Optimized droplet sizing of water-in-crude oil emulsions using nuclear magnetic resonance. Energy Fuels 28(3):1756-1764. https ://doi.org/10.1021/ef402117k

Geim AK, Novoselov KS (2017) The rise of graphene. Chem Rev 114(1):E9793-E9801. https://doi.org/10.1073/pnas.1710996114

Goni GJ, Trinanes JA, MacFadyen A, Streett D, Olascoaga MJ, Imhoff ML et al (2015) Variability of the deepwater horizon surface oil spill extent and its relationship to varying ocean currents and extreme weather conditions. https://doi. org/10.1007/978-3-319-16459-5_1

Goodarzi F, Zendehboudi S (2019) A comprehensive review on emulsions and emulsion stability in chemical and energy industries. Can J Chem Eng 97(1):281-309. https://doi.org/10.1002/ cjce. 23336

Grenoble Z, Trabelsi S (2018) Mechanisms, performance optimization and new developments in demulsification processes for oil and gas applications. Adv Coll Interface Sci 260:32-45. https ://doi.org/10.1016/j.cis.2018.08.003

Griffin WC (1949) Classification of surface-active agents by "HLB." Chicago chapter, pp 311-326. http://www.hindawi.com/journ als/jam/2012/187421/. Accessed 17 July 2019

Hazrati N, Miran Beigi AA, Abdouss M (2018) Demulsification of water in crude oil emulsion using long chain imidazolium ionic liquids and optimization of parameters. Fuel 229(November 2017):126-134. https://doi.org/10.1016/j.fuel.2018.05.010

Israelachvili J (1994) The science and applications of emulsions-an overview. Colloids Surf A: Physicochem Eng Asp 91(C):1-8. https://doi.org/10.1016/0927-7757(94)02743-9

Kokal S, Aramco S (2005) Crude oil emulsions: a state-of-the-art review. SPE Prod Facil 20(December 2004):5-13. https://doi. org/10.2118/77497-PA

Lee RF (1999) Agents which promote and stabilize water-in-oil emulsions. Spill Sci Technol Bull 5(2):117-126. https://doi. org/10.1016/S1353-2561(98)00028-0

Li Y, Zhang Z, Ge B, Men X, Xue Q (2017) A versatile and efficient approach to separate both surfactant-stabilized water-in-oil and oil-in-water emulsions. Sep Purif Technol 176:1-7. https://doi. org/10.1016/j.seppur.2016.11.072

Li Z, Geng H, Wang X, Jing B, Liu Y, Tan Y (2018) Noval tannic acid-based polyether as an effective demulsifier for water-inaging crude oil emulsions. Chem Eng J 354(May):1110-1119. https://doi.org/10.1016/j.cej.2018.08.117

Liu J, Li X, Jia W, Li Z, Zhao Y, Ren S (2015) Demulsification of crude oil-in-water emulsions driven by graphene oxide nanosheets. Energy Fuels 29:4644-4653. https://doi. org/10.1021/acs.energyfuels.5b00966

Liu J, Wang H, Li X, Jia W, Zhao Y, Ren S (2017) Recyclable magnetic graphene oxide for rapid and efficient demulsification

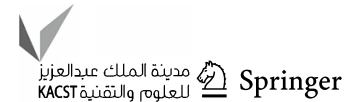


of crude oil-in-water emulsion. Fuel 189:79-87. https://doi. org/10.1016/j.fuel.2016.10.066

López-Chávez E, Pacheco-Sánchez JH, Martínez-Magadán JM, Castillo-Alvarado FDL, Soto-Figueroa C, García-Cruz I (2007) Methodology for predicting the phase envelope of a heavy crude oil and its asphaltene deposition onset. Pet Sci Technol 25(12):19-39. https://doi.org/10.1080/10916460601054180

Martínez-Palou R, Cerón-Camacho R, Chávez B, Vallejo AA, Villanueva-Negrete D, Castellanos J, Aburto J (2013) Demulsification of heavy crude oil-in-water emulsions: a comparative study between microwave and thermal heating. Fuel 113:407-414. https://doi.org/10.1016/j.fuel.2013.05.094

Matijasevic B, Banhart J (2006) Improvement of aluminium foam technology by tailoring of blowing agent. Scripta Mater 54(4):503-508. https://doi.org/10.1016/j.scriptamat .2005 .10 .045

McLean JD, Kilpatrick PK (1997) Effects of asphaltene solvency on stability of water-in-crude-oil emulsions. J Colloid Interface Sci 189(2):242-253. https://doi.org/10.1006/jcis.1997.4807

Mhatre S, Simon S, Sjöblom J, Xu Z (2018) Demulsifier assisted film thinning and coalescence in crude oil emulsions under DC electric fields. Chem Eng Res Des 134:117-129. https://doi.org/10.1016/j. cherd.2018.04.001

Migahed MA, Al-Sabagh AM (2009) Beneficial role of surfactants as corrosion inhibitors in petroleum industry: a review article. Chem Eng Commun 196(9):1054-1075. https://doi.org/10.1080/00986 440902897095

Mohammed RA, Bailey AI, Luckham PF, Taylor SE (1994) Dewatering of crude oil emulsions 3. Emulsion resolution by chemical means. Colloids Surf A: Physicochem Eng Asp 83(3):261-271. https:// doi.org/10.1016/0927-7757(93)02706-K

Mohyaldinn ME, Hassan AM, Ayoub MA (2018) Application of emulsions and microemulsions in enhanced oil recovery and well stimulation. Intech Open, $i$ (tourism), p 13

Mouraille O, Skodvin T, Sjöblom J, Peytavy JL (1998) Stability of water-in-crude oil emulsions: role played by the state of solvation of asphaltenes and by waxes. J Dispers Sci Technol 19(2-3):339367. https://doi.org/10.1080/01932699808913179

Nakama Y (2017) Surfactants. In: Cosmetic science and technology, pp 231-244. https://doi.org/10.1016/B978-0-12-802005-0.00015-X

Nikkhah M, Tohidian T, Rahimpour MR, Jahanmiri A (2015) Efficient demulsification of water-in-oil emulsion by a novel nano-titania modified chemical demulsifier. Chem Eng Res Des 94:164-172. https://doi.org/10.1016/j.cherd.2014.07.021

Pasban AA, Sadeghpour S, Masoumi M, Akbar A, Beigi M (2017) Acidity removal of Iranian heavy crude oils by nanofluid demulsifier: an experimental investigation. J Nano Anal 4(1):112-118

Peña AA, Hirasaki GJ, Miller CA (2005) Chemically induced destabilization of water-in-crude oil emulsions. Ind Eng Chem Res 44(5):1139-1149. https://doi.org/10.1021/ie049666i

Peralta-Martínez MV, Arriola-Medellín A, Manzanares-Papayanopoulos E, Sánchez-Sánchez R, Palacios-Lozano EM (2004) Influence of the speed mixing-on viscosity and droplet size of oil in water emulsions. Pet Sci Technol 22(7-8):1035-1043. https://doi. org/10.1081/LFT-120038709

Porter MR (1991) Handbook of surfactants. Springer, Boston

Salager JL (2002) Surfactants types and uses. FIRP Booklet(E300A), p 2
Salager JL, Forgiarini AM (2012) Emulsion stabilization, breaking, and inversion depends upon formulation: advantage or inconvenience in flow assurance. Energy Fuels 26(7):4027-4033. https:// doi.org/10.1021/ef3001604

Schramm LL, Stasiuk EN, Marangoni DG (2003) Surfactants and their applications. Ann Rep Prog Chem Sect C 99(August):3-48. https ://doi.org/10.1039/B208499F

Sheng J (2010) Modern chemical enhanced oil recovery: theory and practice. Gulf Professional Publishing, Oxford

Sjöblom J (2001) Encyclopedic handbook of emulsion technology. CRC Press, Hoboken

Speight JG (1982) The chemistry and technology of petroleum. Fuel Process Technol 5(3-4):325-326. https://doi.org/10.1016/03783820(82)90026-1

Speight JG (1992) Proceeding of the 4th international conference on stability and handling of liquid fuels. U.S. Department of Energy (DOE/CONF-911102), p 166

Speight JG (2014) The chemistry and technology of petroleum, 5th edn. Fuel Processing Technology. CRC Press, p 953

Tao J, Shi P, Fang S, Li K, Zhang H, Duan M (2015) Effect of rheology properties of oil/water interface on demulsification of crude oil emulsions. Ind Eng Chem Res 54(17):4851-4860. https://doi. org/10.1021/acs.iecr.5b00639

Umar AA, Saaid IBM, Sulaimon AA, Pilus RBM (2018) A review of petroleum emulsions and recent progress on water-in-crude oil emulsions stabilized by natural surfactants and solids. J Pet Sci Eng 165(September 2017):673-690. https://doi.org/10.1016/j. petrol.2018.03.014

Wong SF, Lim JS, Dol SS (2015) Crude oil emulsion: a review on formation, classification and stability of water-in-oil emulsions. J Pet Sci Eng 135:498-504. https://doi.org/10.1016/j.petro 1.2015.10.006

Yang F, Tchoukov P, Qiao P, Ma X, Pensini E, Dabros T et al (2018) Studying demulsification mechanisms of water-in-crude oil emulsions using a modified thin liquid film technique. Colloids Surf A: Physicochem Eng Asp 540(November 2017):215-223. https ://doi.org/10.1016/j.colsurfa.2017.12.056

Zaki NN (1997) Surfactant stabilized crude oil-in-water emulsions for pipeline transportation of viscous crude oils. Colloids Surf A: Physicochem Eng Asp 125(1):19-25. https://doi.org/10.1016/ S0927-7757(96)03768-5

Zaki N, Schoriing PC, Rahimian I (2000) Effect of asphaltene and resins on the stability of water-in-waxy oil emulsions. Pet Sci Technol 18(7-8):945-963. https://doi.org/10.1080/1091646000 8949884

Zolfaghari R, Fakhru'l-Razi A, Abdullah LC, Elnashaie SSEH, Pendashteh A (2016) Demulsification techniques of water-in-oil and oil-in-water emulsions in petroleum industry. Sep Purif Technol 170:377-407. https://doi.org/10.1016/j.seppur.2016.06.026

Publisher's Note Springer Nature remains neutral with regard to jurisdictional claims in published maps and institutional affiliations. 